\title{
Aerosol Nanoencapsulation: Single-Pass Floating Self-Assembly of Biofunctional Hybrid Nanoplatforms
}

\author{
Jeong Hoon Byeon ${ }^{*}$
}

School of Mechanical Engineering, Yeungnam University, Gyeongsan 38541, Republic of Korea

\author{
*E-mail: postjb@yu.ac.kr
}




\section{METHODS}

\section{- Aerosol Nanoencapsulation}

A schematic of the aerosol nanoencapsulation used for these experiments are shown in Figure 1. The obtained nanoplatform is composed of a NP core and an MP shell. Briefly, a spark ablation reactor produced aerosol NPs $\left(\mathrm{SiO}_{2}\right.$ or $\left.\mathrm{Au}\right) . \mathrm{SiO}_{2}$ (or $\left.\mathrm{Au}\right)$ NPs were generated from a spark channel between $\mathrm{Si}$ (or $\mathrm{Au}$ ) rods under air (or nitrogen) flow. $\mathrm{CaCO}_{3} \mathrm{NPs}$ were generated by atomizing-drying, which was achieved with the use of a collison atomizer and diffusion dryer. $0.2 \mathrm{~g}$ of $\mathrm{Ca}\left(\mathrm{HCO}_{3}\right)_{2}$ dissolved solution was atomized with carbon dioxide gas into droplets, and then passed through a diffusion dryer to extract water, as reported by Zhao et al. ${ }^{\mathrm{S} 1}$ For AuG or AuT generation, the spark produced Au NPs passed over a collison atomizer (containing $0.05 \%(w / v)$ graphene nanoflakes or $1.00 \%(v / v) 1$-hexanethiol solution, ethanol-based) orifice where they mixed with atomized particles to form hybrid droplets. The graphene was synthesized by a modified Hummers method ${ }^{\mathrm{S} 2}$ using spark produced graphite particles as the starting material. ${ }^{\mathrm{S} 3}$ The droplets then passed through a diffusion dryer to drive ethanol from the droplets. The gas (air, nitrogen, or carbon dioxide) flow rate, which was controlled by a mass flow controller (3810DS, Kofloc, Japan), was $3 \mathrm{~L} \mathrm{~min}^{-1}$.

While $\mathrm{CaCO}_{3}$ NPs were produced by atomizing-drying, AuT and AuG NPss were produced in the aerosol-state as described in previous reports. ${ }^{\mathrm{S} 4, \mathrm{~S} 5}$ The produced NP-laden $\left(\mathrm{SiO}_{2}, \mathrm{CaCO}_{3}, \mathrm{AuG}\right.$, or AuT) gas flow was used as the operating gas for atomizing the MP solution ${ }^{\mathrm{S} 6}$ containing $0.2 \mathrm{~g}$ of $\mathrm{Ch}-\mathrm{Cs}$ dissolved in $99.8 \mathrm{~mL}$ of $1 \%(v / v)$ with $0.2 \mathrm{~mL}$ of PEI (25 kDa, Sigma-Aldrich, USA). The NPs passed over the atomizer orifice where they mixed with atomized particles to form hybrid droplets. The droplets then passed through a heated tubular flow reactor operating at a $90^{\circ} \mathrm{C}$ wall temperature to drive diluted acetic acid from the droplets. The condition for complete evaporation can be estimated by considering the time required for the evaporation of the droplets and comparing it with the appropriate residence time in the tubular flow reactor. The characteristic time to saturate the gas with vapor from the evaporating droplets, $\tau_{\mathrm{d}}$, is given via the equation, ${ }^{\mathrm{S} 7}$ 
$\tau_{d}=\frac{1}{2 \pi D_{d} \delta_{v} C_{n}\left(D_{d}\right)}$

where $D_{\mathrm{d}}, \delta_{\mathrm{v}}$, and $C_{\mathrm{n}}\left(D_{\mathrm{d}}\right)$ are the droplet diameter, vapor diffusion coefficient, and the droplet number concentration, respectively. The $D_{\mathrm{d}}$ value can be estimated via the equation,

$D_{d}=\left(\frac{\rho_{s} w+\rho_{p}(1-w)}{\rho_{s} w} D_{p}^{3}\right)^{1 / 3}$

where $\rho_{\mathrm{s}}$ and $\rho_{\mathrm{p}}$ are the densities of the solvent and the solid particle, respectively, $w$ is the weight fraction of the solid in liquid, and $D_{\mathrm{p}}$ is the size of the solid particle.

\section{- Instrumentation}

The size distributions of the aerosol particles were measured using a scanning mobility particle sizer (SMPS), consisting of an electrostatic classifier (3085, TSI, USA), condensation particle counter (3776, TSI, USA), and aerosol charge neutralizer (4530, HCT, Korea). The SMPS system, which measures the mobility equivalent diameter, was operated at a sample flow of $0.3 \mathrm{~L} \mathrm{~min}^{-1}$, a sheath flow of $1.0 \mathrm{~L} \mathrm{~min}^{-1}$, and a scan time of $135 \mathrm{sec}$ (measurement range: 7.91-333.8 nm). The mass of the NP@MP $(m)$ was measured using a microbalance (DV215CD, Ohaus, Switzerland) and also estimated via the following equation:

$\left.m=Q \cdot t_{s} \int_{0}^{\infty} \eta\left(D_{p}\right) C_{m}\left(D_{p}\right) d D_{p}\right)$

where $Q$ is the flow rate of nitrogen gas, $t_{\mathrm{s}}$ is the sampling time, $\eta\left(D_{\mathrm{p}}\right)$ is the fractional collection efficiency, and $C_{\mathrm{m}}\left(D_{\mathrm{p}}\right)$ is the mass concentration of particles.

Transmission electron microscope (TEM, CM-100, FEI/Philips, USA) images were obtained at an accelerating voltage of $46-180 \mathrm{kV}$. Specimens were prepared for examination in the TEM by direct electrostatic aerosol sampling at a sampling flow of $0.5 \mathrm{~L} \mathrm{~min}^{-1}$ and an operating voltage of $5 \mathrm{kV}$ using a Nano Particle Collector (NPC-10, HCT, Korea).

For Fourier transform infrared spectroscopy (FTIR) analysis, samples were prepared using 
polytetrafluoroethylene media substrate $(0.2 \mu \mathrm{m}$ pore size, $47 \mathrm{~mm}$ diameter, $11807-47-\mathrm{N}$, Sartorius, Germany) by physical filtration (i.e. mechanical filtration mainly by diffusion, of particles on the surfaces of the substrate), and the spectra were recorded on a Nicolet 6700 FTIR spectrometer (Thermo Electron, USA). The spectra were taken for samples in the range of $4000-400 \mathrm{~cm}^{-1}$ in absorbance mode.

The zeta potential of the NP@MP-gene complexes was determined using a zeta potential analyzer (Nano ZS-90, Malvern Instruments, UK). The NP@MP nanoplatforms were mixed with gene, and incubated at room temperature for $30 \mathrm{~min}$. The complexes were then diluted with double de-ionized water to an appropriate concentration. Measurements of the zeta potential were carried out at $25^{\circ} \mathrm{C}$ and calculated using the manufacturer's supplied software.

\section{- Agarose Gel Retardation Assay}

The gene condensation ability of the NP@MP nanoplatforms under different weight ratios was analyzed by $1 \%$ agarose gel electrophoresis using tris-acetate-ethylenediaminetetraacetic acid buffer (242 g Tris, $57.1 \mathrm{~mL}$ glacial acetic acid, and $0.5 \mathrm{mM}$ ethylenediaminetetraacetic acid, $\mathrm{pH}$ 8.0) containing $0.5 \mu \mathrm{g} \mathrm{m}^{-1}$ ethidium bromide. Complexes containing NP@MP and gene with different weight ratios were prepared by mixing, vortexing, and incubating them at room temperature for 30 min. Approximately $100 \mathrm{ng}$ of each complex was loaded on agarose gels. Gel loading dye blue (New England BioLabs, USA) was added to each well and agarose gel electrophoresis was carried out at a constant voltage of $80 \mathrm{~V}$ for $50 \mathrm{~min}$. The gene bands of the resultant gels were then visualized under a ultraviolet transilluminator at a wavelength of $365 \mathrm{~nm}$.

\section{- In Vitro Cytotoxicity and Transfection}

The cytotoxicity of the NP@MP was evaluated using 293 human embryonic kidney cells by the MTS, 3-(4,5-dimethyl-thiazol-2-yl)-5-(3-carboxymethoxyphenyl)-2-(4-sulfophenyl)2H-tetrazolium, assay. The cells were cultured in $200 \mathrm{~mL}$ of Dulbecco's modified eagle medium (DMEM) supplemented with $10 \%$ fetal bovine serum (FBS) at $37^{\circ} \mathrm{C}, 5 \% \mathrm{CO}_{2}$, and $95 \%$ relative humidity. The cells were seeded in a 96-well microtiter plate (Nunc, Germany) at densities of $1 \times 10^{5}$ cells well ${ }^{-1}$. After $24 \mathrm{~h}$, the culture media were replaced with serum-supplemented culture media containing the NP@MP $\left(1 \mathrm{mg} \mathrm{mL}^{-1}\right)$, and 
the cells were incubated for 24 h. $30 \mu \mathrm{L}$ of the MTS reagent was then added to each well. The cells were incubated for an additional $2 \mathrm{~h}$. The absorbance was then measured using a microplate reader (Spectra Plus, TECAN, Switzerland) at a wavelength of $490 \mathrm{~nm}$. The cell viability (\%) was compared with that of the untreated control cell in media without NP@MP and calculated with $[\mathrm{A}]_{\text {test }}:[\mathrm{A}]_{\text {control }} \times 100 \%$, where $[\mathrm{A}]_{\text {test }}$ is the absorbance of the wells with NP@MP and $[\mathrm{A}]_{\text {control }}$ is the absorbance of the control wells.

The 293 cells were seeded in 24 -well plates at a density of $1 \times 10^{6}$ cells well $^{-1}$ in $1 \mathrm{~mL}$ of complete DMEM medium supplement with $10 \% \mathrm{FBS}$ at $37^{\circ} \mathrm{C}, 5 \% \mathrm{CO}_{2}$, and $95 \%$ relative humidity, one night before transfection. The culture medium was replaced with a serum free DMEM medium, and transfection complexes were added to the cells. The cells were incubated with the transfection complexes at $37^{\circ} \mathrm{C}$ for an additional $24 \mathrm{~h}$ after the medium was replaced by a fresh complete medium. After incubation for $24 \mathrm{~h}$, the medium was aspirated and washed with phosphate-buffered saline. The cells were trypsinized, and the transfection results were then measured by fluorescence activated cell sorting. The GFP plasmid expression of the NP@MP in the 293 cells was then observed with a fluorescent microscope (Nikon Eclipse TE2000-S, USA).

\section{- Photo- and Chemo-Thermal Therapy}

Photo-thermal therapy: Prepared Au-SiO ${ }_{2} @ \mathrm{PM}$ was evenly dispersed in 2\% agar with HeLa cells at concentration of $100 \mu \mathrm{g} \mathrm{mL} \mathrm{m}^{-1}$. The gels were formed in shallow, $35 \mathrm{~mm}$ diameter plastic petri dishes. For exposure, the gel phantom samples at room temparature were exposed to a $705 \mathrm{~nm}$ continuous wave NIR laser beam $\left(1.8 \mathrm{~W} \mathrm{~cm}^{-2}\right.$ in power density) emitted by a solid state laser system (HL7001MG, Opnext, Japan). The gel samples were positioned in the laser beam and irradiated by the beam for fixed duration of $3 \mathrm{~min}$, and then the cells were incubated at $37{ }^{\circ} \mathrm{C}$ with $5 \% \mathrm{CO}_{2}$ for further $24 \mathrm{~h}$. After this treatment, cell viability was determined by a fluorescent microscope after cell staining.

Chemo-thermal Dox release: A hydrophobic substrate-capped reservoir with Au-CaCO $\mathrm{C}_{3} @ \mathrm{Dox}-\mathrm{PM}$ NPs was placed in a PBS flow chamber and irradiated from the top surface with 705-nm NIR laser. The release amount was determined from the absorbance at $480 \mathrm{~nm}$ by UV-vis spectrophotometer (330, 
Perkin-Elmer, USA).

All experiments were performed in triplicate, and the results were reported as means and standard deviations. Statistical analyses were performed using Student's $t$-test. The differences were considered significant for $p<0.05$. 
FIGURE S1
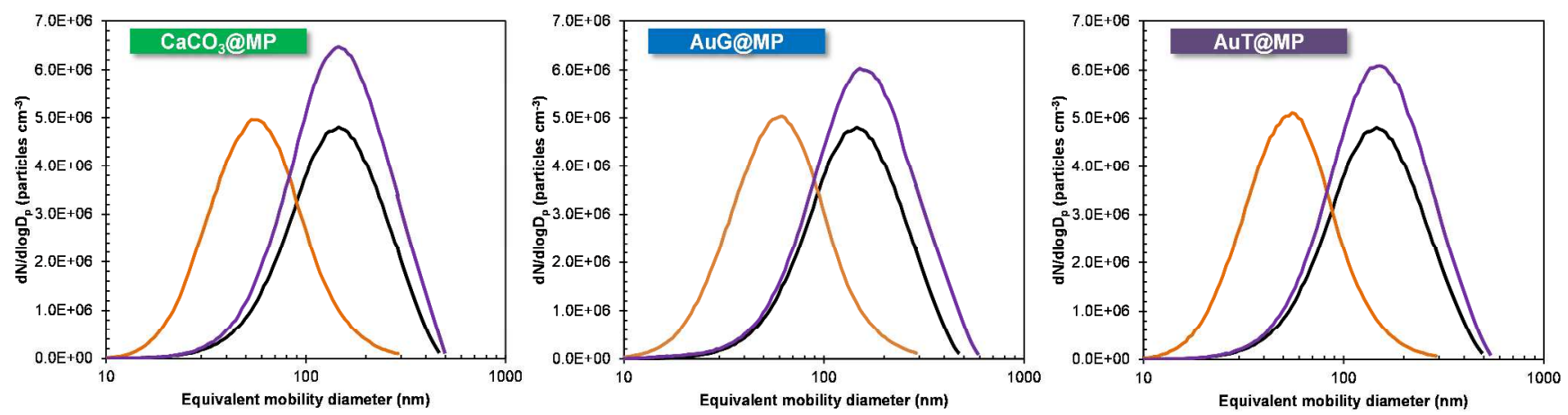

-: MP

- NP

-: NP@MP

Figure S1. Size distributions of aerosol MP particles alone, other NPs $\left(\mathrm{CaCO}_{3}, \mathrm{AuG}\right.$, or $\left.\mathrm{AuT}\right)$, and MPencapsulated NPs $\left(\mathrm{CaCO}_{3} @, \mathrm{AuG} @\right.$, or AuT@MP). 
FIGURE S2



Figure S2. FTIR spectra of MP particles alone, and MP-encapsulated NPs $\left(\mathrm{SiO}_{2} @, \mathrm{CaCO}_{3} @, \mathrm{AuG} @\right.$, orAuT@MP). 
FIGURE S3



In vitro cytotoxicity of MP particles alone, and MP-encapsulated NPs $\left(\mathrm{SiO}_{2} @, \mathrm{CaCO}_{3} @, \mathrm{AuG} @\right.$, or AuT@MP) in the 293 cells at different mass concentrations $\left(5-100 \mu \mathrm{g} \mathrm{mL} \mathrm{L}^{-1}\right)$. 
TABLE S1 Size distributions of aerosol MP particles alone, individual $\mathrm{NPs}\left(\mathrm{SiO}_{2}, \mathrm{CaCO}_{3}, \mathrm{AuG}\right.$, or AuT), and MP-encapsulated NPs $\left(\mathrm{SiO}_{2} @, \mathrm{CaCO}_{3} @\right.$, AuG@, or AuT@MP)

\begin{tabular}{|c|c|c|c|}
\hline Case & Mean Diameter (nm) & Standard Deviation (-) & $\begin{array}{l}\text { Number Concentration } \\
\left(\text { particles } \mathrm{cm}^{-3}\right)\end{array}$ \\
\hline MP & 130 & 1.86 & $2.7 \times 10^{6}$ \\
\hline \multicolumn{4}{|c|}{$\mathrm{SiO}_{2} @ \mathrm{MP}$} \\
\hline $\mathrm{SiO}_{2}$ & 57 & 1.75 & $2.8 \times 10^{6}$ \\
\hline $\mathrm{SiO}_{2} @ \mathrm{MP}$ & 130 & 1.85 & $3.4 \times 10^{6}$ \\
\hline \multicolumn{4}{|c|}{$\mathrm{CaCO}_{3} @ \mathrm{MP}$} \\
\hline $\mathrm{CaCO}_{3}$ & 57 & 1.75 & $2.9 \times 10^{6}$ \\
\hline $\mathrm{CaCO}_{3} @ \mathrm{MP}$ & 130 & 1.86 & $4.0 \times 10^{6}$ \\
\hline \multicolumn{4}{|c|}{ AuG@MP } \\
\hline AuG & 60 & 1.75 & $2.9 \times 10^{6}$ \\
\hline AuG@MP & 135 & 1.90 & $3.5 \times 10^{6}$ \\
\hline \multicolumn{4}{|c|}{ AuT@MP } \\
\hline AuT & 54 & 1.70 & $2.9 \times 10^{6}$ \\
\hline AuT@MP & 135 & 1.85 & $3.5 \times 10^{6}$ \\
\hline
\end{tabular}

TABLE S2 Zeta potential of gene complexes with MP particles alone, and MP-encapsulated NPs $\left(\mathrm{SiO}_{2} @, \mathrm{CaCO}_{3} @, \mathrm{AuG} @\right.$, or AuT@MP)

\section{Platforms-Gene Complexes}

\begin{tabular}{l}
\hline $\mathrm{MP}$ \\
\hline $\mathrm{SiO}_{2} @ M P$ \\
\hline $\mathrm{CaCO}_{3} @ M P$ \\
\hline $\mathrm{AuG} @ \mathrm{MP}$ \\
\hline $\mathrm{AuT} @ \mathrm{MP}$
\end{tabular}

\section{Zeta Potential $(\mathrm{mV})$}

$19.8 \pm 3.91$

$20.7 \pm 4.85$

$24.4 \pm 3.25$

$22.3 \pm 2.67$

$27.2 \pm 3.60$ 


\section{SUPPORTING REFERENCES}

(S1) Zhao, D. F.; Buchholz, A.; Mentel, Th. F.; Müller, K. -P.; Borchardt, J.; Kiendler-Scharr, A.;

Spindler, C.; Tillmann, R.; Trimborn, A.; Zhu, T.; Wahner, A. Novel Method of Generation of $\mathrm{Ca}\left(\mathrm{HCO}_{3}\right)_{2}$ and $\mathrm{CaCO}_{3}$ Aerosols and First Determination of Hygroscopic and Cloud Condensation Nuclei Activation Properties. Atmos. Chem. Phys. 2010, 10, 8601-8616.

(S2) Hummers, W. S.; Offerman, R. E. Preparation of Graphitic Oxide. J. Am. Chem. Soc. 1958, 80, 1339-1339.

(S3) Byeon, J. H.; Park, J. H.; Yoon, K. Y.; Ko, B. J.; Ji, J. H.; Hwang, J. Removal of Volatile Organic Compounds by Spark Generated Carbon Aerosol Particles. Carbon 2006, 44, 2106-2108.

(S4) Byeon, J. H.; Roberts, J. T. Aerosol Based Fabrication of Thiol-Capped Gold Nanoparticles and Their Application for Gene Transfection. Chem. Mater. 2012, 24, 3544-3549.

(S5) Byeon, J. H.; Kim, Y.-W. Continuous Gas-Phase Synthesis of Graphene Nanoflakes Hybridized by Gold Nanocrystals for Efficient Water Purification and Gene Transfection. Chem. Eng. J. 2013, 229, 540-546.

(S6) Byeon, J. H.; Kim, H. -K.; Thompson, D. H.; Roberts, J. T. Aerosol-Based Fabrication of Modified Chitosans and Their Application for Gene Transfection. ACS Appl. Mater. Interfaces 2014, 6, 45974602.

(S7) Byeon, J. H.; Kim, H.-K.; Roberts, J. T. Monodisperse Poly(lactide-co-glycolic acid)-Based Nanocarriers for Gene Transfection. Macromol. Rapid Commun. 2012, 33, 1840-1844. 